Pacific Journal of Mathematics

ALGEBRAS WITH MINIMAL LEFT IDEALS WHICH ARE 


\title{
ALGEBRAS WITH MINIMAL LEFT IDEALS WHICH ARE HILBERT SPACES
}

\author{
BRUCE A. BARNES
}

\begin{abstract}
This paper gives a necessary and sufficient condition that certain topological algebras $A$ (normed algebras and algebras which are inner product spaces) be left (right) annihilator algebras. It is assumed that the socle of $A$ is dense in $A$ and that a proper involution $*$ is defined on the socle. Then the necessary and sufficient condition is essentially that the minimal left (right) ideals of $A$ be complete in the norm on $A$ and be a Hilbert space in an equivalent norm.
\end{abstract}

We prove a useful preliminary result in $\S 2$. In $\S 3$ we deal with the question of when a normed algebra $A$ is a left or right annihilator algebra. In $\S 4$ we consider this same question when $A$ is a topological algebra in a topology defined by an inner product. This section is motivated by the work of P. Saworotnow and B. Yood on such algebras (see, for example, [5] and [9]). In the final section we generalize the well known result of Bonsall and Goldie that $B^{*}$ annihilator algebras are dual.

Notation and terminology. $A$ is always a complex algebra. $S_{A}$ denotes the socle of $A$, when this exists. If $E$ is a subset of $A$, let $L(E)$ and $R(E)$ denote the left and right annihilator of $E$ respectively $(L(E)=\{u \in A \mid u v=0$ for all $v \in E\})$. $A$ is a left (right) annihilator algebra if for every proper closed right (left) ideal $M$ of $A L(M) \neq 0(R(M) \neq 0)$ and $L(A)=0(R(A)=0)$. A left (right) ideal $M$ of $A$ is a left (right) annihilator ideal if $M=L(E)(M=R(E))$ for some subset $E$ of $A$. If $A$ is semi-simple, $A$ is a modular annihilator algebra if $A / S_{A}$ is a radical algebra; see [8]. Annihilator and dual algebras are defined and discussed in [4, pp.96-107].

An involution * defined on $A$ (or $S_{A}$ ) is proper if $u u^{*}=0$ implies $u=0 . \quad u$ is a self-adjoint if $u=u^{*}$. We denote the set of all selfadjoint minimal idempotents of $A$ by $H$. If $*$ is proper on $S_{A}$, then minimal left (right) ideals of $A$ will have the form $A h(h A), h \in H$, by [4, Lemma 4.10.1, p. 261].

Let $\mathscr{\mathscr { C }}$ be a Hilbert space: $\mathscr{B}(\mathscr{H})$ is the algebra of all bounded operators on $\mathscr{H}, \mathscr{F}(\mathscr{H})$ is the subalgebra of $\mathscr{B}(\mathscr{H})$ consisting of all operators which have finite dimensional range, and $\mathscr{C}(\mathscr{H})$ is the algebra of compact operators on $\mathscr{\mathscr { C }}$. If $T \in \mathscr{B}(\mathscr{L})$, we denote the operator bound of $T$ as $|T|$. Given $u, v \in \mathscr{H}$, we define an operator $(u \mid v)$ on $\mathscr{C}$ by $(u \mid v)(w)=(w, u) v$ for all $w \in \mathscr{l}$. More generally 
if $X$ is a normed linear space and $X^{\prime}$ is the normed dual of $X$, given $x \in X$ and $f \in X^{\prime}$ we define an operator $(f \mid x)$ on $X$ by $(f \mid x)(y)=f(y) x$ for all $y \in X$.

2. Preliminary results. Let $\mathscr{H}$ be a Hilbert space. Assume that $B$ is a subalgebra of $\mathscr{B}(\mathscr{C})$ with $\mathscr{F}(\mathscr{C}) \subset B$. Furthermore, assume that $B$ is a topological linear space with a topology $\mathscr{T}$ such that

(i) The maps $x \rightarrow x y$ and $x \rightarrow y x$ are continuous on $B$ for all $y \in B$;

(ii) $\mathscr{F}(\mathscr{H})$ is dense in $B$ in the topology $\mathscr{T}$;

(iii) If $\left\{u_{n}\right\} \subset \mathscr{H}$ and $u_{n} \rightarrow 0$ in $\mathscr{H}$, then $\left(w \mid u_{n}\right) \rightarrow 0$ in $\mathscr{T}$ for any $w \in \mathscr{H}$.

For $\mathscr{K}$ a closed subspace of $\mathscr{H}$, define

$$
\mathscr{R}(\mathscr{K})=\{T \in B \mid T(\mathscr{H}) \subset \mathscr{K}\} \text {. }
$$

Theorem 2.1. Assume $B$ is as given above. Then $B$ is a left annihilator algebra. Also every right annihilator ideal of $B$ is of the form $\mathscr{R}(\mathscr{K})$ for some closed subspace $\mathscr{K}$ of $\mathscr{H}$. If $T \in \overline{T B}$ for all $T \in B$, then every closed right ideal of $B$ is a right annihilator ideal.

Proof. Assume that $N$ is a closed right ideal of $B$. Let

$$
\mathscr{J}=\{T u \mid T \in N, u \in \mathscr{H}\} \text {. }
$$

Assume that $w=T(u)+S(v)$ where $u, v \in \mathscr{C}$ and $T, S \in N$. Assume that $u \neq 0$, and let $\lambda=|u|_{2}^{2}\left(|\cdot|_{2}\right.$ the norm on $\left.\mathscr{C}\right)$. Then

$$
(1 / \lambda) S(u \mid v) \in N
$$

and $(T+(1 / \lambda) S(u \mid v))(u)=w$. This proves that $\mathscr{J}$ is a subspace of $\mathscr{H}$. Let $\mathscr{K}=\overline{\mathscr{J}}$. The proof of [4, Lemma 2.8.24, p. 104] implies that $R(L(N))=\mathscr{R}(\mathscr{K})$. If $v \in \mathscr{K}$, then there exists $\left\{u_{n}\right\} \subset \mathscr{H}$ and $\left\{T_{n}\right\} \subset N$ such that $T_{n}\left(u_{n}\right)=v_{n} \rightarrow v$ in $\mathscr{H}$. Then given any $w \in \mathscr{H}$, $\left(w \mid v_{n}\right)=T_{n}\left(w \mid u_{n}\right) \in N$ for all $n$. By (iii) $\left(w \mid v_{n}\right) \rightarrow(w \mid v)$ in the topology $\mathscr{T}$. Thus whenever $v \in \mathscr{K}$ and $w \in \mathscr{H},(w \mid v) \in N$. Using this result, the proof of [4, Lemma 2.8.26, p. 105] implies that

$$
R(L(N)) \cdot B \subset N \text {. }
$$

Therefore if $L(N)=0, B^{2} \subset N$, and it follows that $\mathscr{F}(\mathscr{H}) \subset N$. Then by (ii) $N=B$. This proves that $B$ is a left annihilator algebra.

If $T \in \overline{T B}$ for all $T \in B$, then whenever $T \in R(L(N)), T \in \overline{T B} \subset$ $\overline{R(L(N)) \cdot B} \subset N$. Therefore $N=R(L(N))$, so that $N$ is a right annihilator ideal. 
A theorem similar to Theorem 2.1 can be proved concerning the left ideals of $B$. Assume that $\mathscr{F}(\mathscr{H}) \subset B \subset \mathscr{B}(\mathscr{H})$ and that $B$ satisfies (i), (ii), and

(iv) If $\left\{u_{n}\right\} \subset \mathscr{H}$ and $u_{n} \rightarrow 0$ in $\mathscr{H}$, then $\left(u_{n} \mid v\right) \rightarrow 0$ in the topology $\mathscr{T}$ on $B$ for all $v \in \mathscr{H}$

Define $B^{*}=\left\{T^{*} \mid T \in B\right\}$. Topologize $B^{*}$ with the topology

$$
\mathscr{T}^{*}=\left\{U^{*} \mid U \in \mathscr{T}\right\} \text {. }
$$

Then $\mathscr{F}(\mathscr{H}) \subset B^{*} \subset \mathscr{B}(\mathscr{C})$ and $B^{*}$ satisfies (i) and (ii). But also by (iv) and the fact that $(v \mid w)^{*}=(w \mid v)$ for all $v, w \in \mathscr{C}, B^{*}$ satisfies (iii). Then the conclusions of Theorem 2.1 hold for $B^{*}$. Therefore $B^{*}$ is a left annihilator algebra and every right annihilator ideal is of the form $\left\{T \in B^{*} \mid T(\mathscr{C}) \subset \mathscr{K}\right\}$ for some closed subspace $\mathscr{K}$ of $\mathscr{H}$. Let $N$ be a proper closed left ideal of $B$. Then $N^{*}$ is a proper closed right ideal of $B^{*}$. Therefore there exists $T \in B, T \neq 0$, such that $T^{*} N^{*}=0$. Then $R(N) \neq 0$. Now assume that $N$ is a left annihilator ideal of $B$. Then $N^{*}$ is a right annihilator ideal of $B^{*}$ which implies that $N^{*}=\left\{T \in B^{*} \mid T(\mathscr{H}) \subset \mathscr{K}^{\perp}\right\}$ for $\mathscr{K}$ some closed subspace of $\mathscr{K}$. Then it is not difficult to verify that

$$
N=\{T \in B \mid T(\mathscr{K})=0\} \text {. }
$$

Finally if $T \in \overline{B T}$ for all $T \in B$, then $T \in \overline{T B^{*}}$ for all $T \in B^{*}$. This implies that when $T \in \overline{B T}$ for all $T \in B$, then every closed left ideal of $B$ is a left annihilator ideal (by Theorem 2.1 again).

Combining these remarks and Theorem 2.1 we have the following result.

Theorem 2.2. Assume that $\mathscr{F}(\mathscr{H}) \subset B \subset \mathscr{B}(\mathscr{P})$ and that $B$ satisfies (i)-(iv). Then $B$ is an annihilator algebra. If in addition $T \in \overline{T B}$ and $T \in \overline{B T}$ for all $T \in B$, then $B$ is dual.

3. Normed algebras. We assume throughout this section that $A$ is a semi-simple modular annihilator algebra, that there is a proper involution * defined on $S_{A}$, and that $A$ is a normed algebra with norm $\|\cdot\|$. Recall that $H$ denotes the set of self-adjoint minimal idempotents of $A$. When $h \in H$, we define a functional $f_{h}$ on $S_{A}$ by the rule $f_{h}(u) h=h u h$. By the proof of $[7$, Th. 5.2, p. 358] we have that $f_{h}$ is a positive hermitian functional on $S_{A}$. We introduce an inner product on the minimal left ideal $A h$ by the usual definition, $(u h, v h)=f_{h}((v h) * u h), u, v \in A$. We call this inner product the cannonical inner product on $A h$ and denote the corresponding norm by 
$\left.1 \cdot\right|_{2}$. We define a ${ }^{*}$-representation of $S_{A}$ on the inner product space $A h$ by $u \rightarrow T_{u}^{h}, u \in S_{A}$, where $T_{u}^{h}(v h)=u v h$ for all $v \in A$. As shown in the proof of $\left[7, T h .5 .2\right.$, p. 358], the operators $T_{u}^{h}$ are bounded on $A h$. Also by [7, Lemma 7.1, p. 358] $T_{u}^{h}$ has finite dimensional range on $A h$ for all $u \in S_{A}$. In a similar fashion a cannonical inner product can be introduced on the minimal right ideal $h A$, and a *-representation of $S_{A}$ can be constructed into $\mathscr{B}(h A)$.

Since $S_{A}$ is a modular annihilator algebra with proper involution *, then by $\left[1,(1.3)\right.$, p. 6] there is a unique norm $|\cdot|$ on $S_{A}$ with the property that $\left|u u^{*}\right|=|u|^{2}$ for all $u \in S_{A}$. We call $|\cdot|$ the operator norm on $S_{A}$.

Theorem 3.1. Assume that $A$ is a left (right) annihilator algebra in the norm $\|\cdot\|$. Also assume that there exists $K>0$ such that $K\|u\| \geqq|u|$ for all $u \in S_{A}$. Then for any $h \in H, A h(h A)$ is a Hilbert space in the cannonical norm $|\cdot|_{2}$, and $\|\cdot\|$ and $|\cdot|_{2}$ are equivalent on $A h(h A)$.

Proof. We consider only the case where $A$ is a left annihilator algebra. Also it is sufficient to prove the theorem when $A$ is primitive. For in the general case given $h \in H, A h$ is a minimal left ideal of some minimal closed two sided ideal $M$ of $A$. Then $M$ is primitive and by the proof of [4, Th. 2.8.12, p. 99] $M$ is a left annihilator algebra. Therefore assume that $A$ is primitive. We shall show that $S_{A}$ is a left annihilator algebra. If $N$ is a proper closed right ideal of $S_{A}$, then $\bar{N}$, the closure of $N$ in $A$, is a proper closed right ideal of $A$. Then $L(\bar{N}) \neq 0$, and therefore there exists a minimal idempotent $e \in L(\bar{N})$. Then $e \in S_{A}$ and $e N=0$. Thus $S_{A}$ is a left annihilator algebra.

Assume $h \in H$. Note that $|u h|^{2}=\left|(u h)^{*} u h\right|=|u h|_{2}^{2}|h|=|u h|_{2}^{2}$ so that $|\cdot|$ and $|\cdot|_{2}$ coincide on $A h$. By hypothesis $K\|u\| \geqq|u|$ for all $u \in S_{A}$, and therefore $K\|u h\| \geqq|u h|_{2}$ for all $u \in A$. We prove that $\|\cdot\|$ and $|\cdot|_{2}$ are equivalent on $A h$. Since $A$ is primitive, the representation $u \rightarrow T_{u}^{h}$ of $S_{A}$ on $A h$ is faithful. Let $\mathscr{F}=\left\{T_{u}^{h} \mid u \in S_{A}\right\}$. By the proof of $[4$, Lemma 2.8.20, p. 101] $(f \mid x) \in \mathscr{F}$ whenever $f$ is a continuous linear functional on $A h$ with respect to $\|\cdot\|$ and $x \in A h$. It follows that any such functional $f$ must be continuous on $A h$ with respect to $|\cdot|_{2}$. Let $V$ be the normed dual of $A h$ with respect to $\|\cdot\|$, and let $B$ be the unit ball in $A h$ with respect to $|\cdot|_{2}$. For any $f \in V, \sup _{x \in B}|f(x)|<+\infty$. Then by the Uniform Boundedness Theorem applied to the set $B$; $\sup _{x \in B} \sup _{|| f|| \leqq 1}|f(x)| \leqq J$ for some finite number $J$. It follows that $\|x\| \leqq J|x|_{2}$ for all $x \in A h$. Therefore $\|\cdot\|$ and $|\cdot|_{2}$ are equivalent on $A h$.

It remains to be shown that $A h$ is a Hilbert space in the norm 
$|\cdot|_{2}$. Since $K\|u\| \geqq|u|$ for all $u \in S_{A}, S_{A}$ is a left annihilator algebra with respect to $|\cdot|$. Let $\mathscr{H}$ be the Hilbert space completion on $A h$. Given $w \in \mathscr{H}$, we define $f^{\prime}(x)=(x, w)$ for $x \in \mathscr{H}$. Choose $u h \in A h$ such that $|u h|_{2}=1 . \quad(f \mid u h) \in \mathscr{F}$ by the proof of [4, Lemma 2.8.20, p. 101]. Therefore $(f \mid u h)^{*} \in \mathscr{F}$. For any $x \in A h$,

$$
\begin{gathered}
(x, w)=((f \mid u h) x, u h)=\left(x,(f \mid u h)^{*} u h\right) . \text { Therefore } \\
w=(f \mid u h)^{*}(u h) \in A h .
\end{gathered}
$$

Thus $\mathscr{H}=A h$.

Using the previous result, we give an example of a norm on $\mathscr{F}(\mathscr{H})$ in which $\mathscr{F}(\mathscr{H})$ is not a left annihilator algebra. Let $\mathscr{H}$ be an infinite dimensional Hilbert space and denote the norm on $\mathscr{H}$ by $|\cdot|_{2}$. Let $\|\cdot\|$ be any norm on $\mathscr{C}$ such that $|x|_{2} \leqq\|x\|$ for all $x \in \mathscr{H}$, and $|\cdot|_{2}$ and $\|\cdot\|$ are inequivalent on $\mathscr{H}$. If $f$ is any discontinuous linear functional on $\mathscr{H}$, then $\|x\|=|x|_{2}+|f(x)|$ is an example of such a norm. Every functional on $\mathscr{C}$ continuous with respect to $|\cdot|_{2}$ is continuous with respect to $\|\cdot\|$. It follows that every operator $T \in \mathscr{F}(\mathscr{H})$ is bounded in the norm

$$
\|T\|=\sup _{\|x\| \leqq 1}\|T x\| \text {. }
$$

We note that there exists $K>0$ such that $K\|T\| \geqq|T|(|\cdot|$ the operator norm on $\mathscr{F}(\mathscr{H})$ ) by [4, Th. 2.4.17, p. 69]. Now fix $u \in \mathscr{H}$ such that $|u|_{2}=1$. Let $N$ be the minimal left ideal of $\mathscr{F}(\mathscr{H})$ defined by $N=\{(u \mid v) \mid v \in \mathscr{H}\} . \quad v \rightarrow(u \mid v)$ is an isometry of $\mathscr{H}$ in the norm $|\cdot|_{2}$ onto $N$ in the operator norm since $|(u \mid v)|=|u|_{2}|v|_{2}=|v|_{2}$. To verify that $\mathscr{F}(\mathscr{C})$ is not a left annihilator algebra in the norm $\|\cdot\|$, it is sufficient to prove that the map $v \rightarrow(u \mid v)$ is a bicontinuous map from $\mathscr{H}$ in the norm $\|\cdot\|$ onto $N$ in the norm $\|\cdot\|$. For then $\|\cdot\|$ and $|\cdot|$ are inequivalent on $N$, and therefore Theorem 3.1 gives the result.

$$
\|(u \mid v)\|=\sup _{\|x\| \leqq 1}\|(u \mid v)(x)\|=\sup _{\|x\| \mid \leqq 1}|(x, u)\|\mid v\| \leqq\|v\|,
$$

and

$$
\|(u \mid v)\| \geqq\|(u \mid v)(u /\|u\|)\|=(1 /\|u\|)\|v\| .
$$

This completes the example.

Now we prove a converse of Theorem 3.1.

Theorem 3.2. Assume that $S_{A}$ is dense in A. Assume that for every $h \in H$ Ah $(h A)$ is a Hilbert space in the norm $|\cdot|_{2}$, and that $|\cdot|_{2}$ and $\|\cdot\|$ are equivalent on $A h(h A)$. Then $A$ is a left (right) 
annihilator algebra. If in addition $u \in \overline{u A}(u \in \overline{A u})$ for all $u \in A$, then every closed right (left) ideal of $A$ is a right (left) annihilator ideal.

Proof. We assume that for every $h \in H A h$ is a Hilbert space in the norm $|\cdot|_{2}$, and that $|\cdot|_{2}$ and $\|\cdot\|$ are equivalent on $A h$. First suppose that $A$ is primitive. Given $h \in H$, then $u \rightarrow T_{u}^{h}$ is a faithful *-representation of $S_{A}$ on the Hilbert space $A h$. Given any $u, v$, $w \in A, T_{(u h)(v h) *}^{h}(w h)=(w h, v h)(u h)=(v h \mid u h)(w h)$. Therefore all the operators of the form $(v h \mid u h)$ are in the image of the representation $w \rightarrow T_{w}^{h}$. It follows that $\mathscr{F}(A h)$ is in the image of this representation. By [4, Th. 2.4.17, p. 69] there exists $K>0$ such that $K\|u\| \geqq\left|T_{u}^{h}\right|$ for all $u \in S_{A}$. Then since $S_{A}$ is dense in $A$, there is a unique extension of the representation $u \rightarrow T_{u}^{h}$ of $S_{A}$ to a representation $u \rightarrow T_{u}$ of $A$ onto a subalgebra $B$ of $\mathscr{B}(A h)$. Therefore

$$
\mathscr{F}(A h) \subset B \subset \mathscr{B}(A h) \text {. }
$$

We consider $B$ normed by $\|\cdot\|$ in the natural way, $\left\|T_{u}\right\|=\|u\|$ for $u \in A . \quad B$ clearly has properties (i) and (ii) listed previous to Theorem 2.1. If $\left|u_{n} h\right|_{2} \rightarrow 0$, then by hypothesis $\left\|u_{n} h\right\| \rightarrow 0$, and therefore

$$
\left\|\left(w h \mid u_{n} h\right) H=\right\| T_{\left(u_{n} h\right)(w h) *} \| \rightarrow 0
$$

for any $w \in A$. This proves that $B$ also satisfies (iii). By Theorem 2.1, $B$, and hence $A$, is a left annihilator algebra. If in addition $u \in \overline{u A}$ for all $u \in A$, then again by Theorem 2.1 , every closed right ideal of $A$ is a right annihilator ideal. This proves the theorem when $A$ is primitive. In the general case let $\left\{M_{\alpha} \mid \alpha \in I\right\}$ be the set of all minimal closed two sided ideals of $A . M_{\alpha}$ is primitive for each $\alpha \in I$, and therefore the theorem holds for each $M_{\alpha}$. Since $A$ has dense socle, $A$ is the topological sum of the $M_{\alpha}, \alpha \in I$. Then by the proof of $[4$, Th. 2.8.29, p. 106], the theorem holds for $A$.

4. Algebras which are inner product spaces. Throughout this section we assume that $A$ is a semi-simple modular annihilator algebra which is an inner product space with inner product $(\cdot, \cdot)$. Also we assume that the maps $x \rightarrow x y$ and $x \rightarrow y x$ are continuous on $A$ for all $y \in A$. An element $x$ has a left (right) adjoint if there exists $w \in A$ such that $(x y, z)=(y, w z)((y x, z)=(x, z w))$ for all $y, z \in A$. If $x \in A$ has a left (right) adjoint, then it is unique. Assume that every element $u \in S_{A}$ has a left adjoint which we denote by $u^{*}$. Suppose that $u^{*} u=0$. By $[1,(2.2)$, p. 6] there exists an idempotent $e \in A$ such that $u=u e$. Then $0=\left(u^{*} u, e\right)=(u, u)$ so that $u=0$. This verifies that $*$ must be proper on $S_{A}$. Similarly if every element in 
$S_{A}$ has a right adjoint, then this adjoint must be proper on $S_{A}$. We denote the norm determined on $A$ by the inner product by $|\cdot|_{2}$.

TheOREM 4.1. Assume that every element $u \in S_{A}$ has a left (right) adjoint $u^{*}$ and that $A$ is a left (right) annihilator algebra. Then for every $h \in H, A h(h A)$ is a Hilbert space in the norm $|\cdot|_{2}$, and $|\cdot|_{2}$ and $|\cdot|_{2}$ are equivalent on $A h(h A)$.

Proof. We prove the "left" part of the theorem only. As in the proof of Theorem 3.1, it is sufficient to prove the theorem when $A$ is primitive. Therefore assume $A$ is primitive. Given $h \in H$,

$$
(u h, v h)=\left((v h)^{*} u h, h\right)=(u h, v h)|h|_{2}^{2}
$$

for all $u, v \in A$. Therefore $|\cdot|_{2}$ and $|\cdot|_{2}$ are equivalent on $A h . \quad u \rightarrow$ $T_{u}^{h}$ is a faithful representation of $S_{A}$ on $A h$. Let $\mathscr{F}=\left\{T_{u}^{h} \mid u \in S_{A}\right\}$. By the same argument as in the proof of Theorem 3.1, $S_{A}$ is a left annihilator algebra with respect to $|\cdot|_{2}$. Then by the proof of [4, Lemma 2.8.20, p. 101] $(f \mid u h) \in \mathscr{F}$ for all $u \in A$ and all functionals $f$ continuous on $A h$ with respect to $|\cdot|_{2}$. Then the argument in the last paragraph of the proof of Theorem 3.1 implies that $A h$ is a Hilbert space in the norm $|\cdot|_{2}$.

Now we prove a result in the other direction.

THEOREM 4.2. Assume that every element $u \in S_{A}$ has a left (right) adjoint $u^{*}$. Assume that $A$ has dense socle in the norm $\mid \cdot l_{2}$ and that for every $h \in H, A h(h A)$ is a Hilbert space in the norm $|\cdot|_{2}$. Then $A$ is a left (right) annihilator algebra. If in addition $u \in \overline{u A}$ $(u \in \overline{A u})$ for all $u \in A$, then every closed right (left) ideal of $A$ is a right (left) annihilator ideal.

Proof. We prove the "left" part of the theorem only. It is sufficient to prove that the theorem holds for each minimal closed two sided ideal $M$ of $A$. For then by the proof of [4, Th. 2.8.29, p. 106] the result follows for $A$. Therefore assume that $M$ is a minimal closed two sided ideal of $A$. Choose $h \in H \cap M$. Then $u \rightarrow$ $T_{u}^{h}$ is a faithful representation of $M$ on the Hilbert space $A h . T_{u}^{h}$ is a bounded operator on $A h$ since $u \rightarrow u x$ is a continuous map on $A$. Let $B=\left\{T_{u}^{h} \mid u \in M\right\}$. We norm $B$ by $\left|T_{u}^{h}\right|_{2}=|u|_{2}$ for $u \in M$, Given $u h$ and $v h$, then $T_{(u h)(v h)^{*}}^{h} \in B$, and

$$
T_{(u h)(v h) *}(w h)=(w h, v h) u h=(v h \mid u h)(w h)
$$

for all $w h \in A h$. Therefore $\mathscr{F}(A h) \subset B . \quad B$ satisfies properties (i) 
and (ii) given previous to Theorem 2.1 by hypothesis. Also as noted in the proof of Theorem 4.1, $|u h|_{2}^{2}=|u h|_{2}^{2}|h|_{2}^{2}$ for all $u \in A$. Therefore if $\left|u_{n} h\right|_{2} \rightarrow 0$, then $\left|u_{n} h\right|_{2} \rightarrow 0$, so that for any $v \in A,\left|u_{n} h(v h)^{*}\right|_{2} \rightarrow$ 0 . It follows that $\left|\left(v h \mid u_{n} h\right)\right|_{2}=\left|T_{\left(u_{n} h\right)(v h) *}^{h}\right|_{2} \rightarrow 0$. Therefore $B$ satisfies (iii). Then Theorem 2.1 applies and this completes the proof.

We apply the previous theorems to right-modular complemented algebras as defined by B. Yood [9, p. 261]. Let $A$ be an algebra with an inner product $(\cdot, \cdot) . A$ is a right-modular complemented algebra if

(a) the maps $x \rightarrow x y$ and $x \rightarrow y x$ are continuous for all $y \in A$,

(b) any right or left ideal $I$ for which $I^{\perp}=\{0\}$ is dense in $A$ (where $I^{\perp}=\{x \in A \mid(x, y)=0$ for all $y \in I\}$ ),

(c) the intersection of the closed modular maximal right ideals of $A$ is $\{0\}$, and $M^{\perp}$ is a right ideal for each closed modular maximal right ideal $M$.

We prove the following theorem.

THEOREM 4.3. Assume that $A$ is a modular annihilator algebra and a right-modular complemented algebra. Then $A$ is an annihilator algebra if and only if every minimal left or right ideal of $A$ is a Hilbert space in the norm determined by the inner product.

Proof. First note that $A$ is semi-simple by property (c). Since $A$ is a modular annihilator algebra, then by [8, Lemma 3.3, p. 38] every modular maximal right ideal $M$ of $A$ is of the form $(1-e) A$ where $e$ is a minimal idempotent of $A$. Then by (a) $M$ is closed. Similarly every modular maximal left ideal of $A$ is closed. Also by [9, Th. 2.1, p. 262] $K^{\perp}$ is a right (left) ideal for all right (left) ideals $K$ of $A$.

Assume that every minimal left or right ideal of $A$ is a Hilbert space in the norm determined by the inner product. Given $K$ a minimal right ideal of $A$, then $N=K^{\perp}$ is a right ideal. Also $N+K$ is dense by (b). Since $K$ is complete, it follows that $N+K=A$. Therefore $N$ is a modular maximal right ideal of $A$. By the proof of [8, Th. 4.5, p. 44] every element of $N^{\perp}=K$ has a left adjoint. Since $K$ was an arbitrary minimal right ideal, then every element in $S_{A}$ has a left adjoint. A similar proof shows that every element of $S_{A}$ has a right adjoint. $A$ has dense socle by (b). Therefore by Theorem 4.2, $A$ is an annihilator algebra.

Now assume that $A$ is an annihilator algebra. Take $K$ minimal right ideal of $A$. Then $N=K^{\perp}$ is a proper closed right ideal of $A$. Since $A$ is an annihilator algebra, there exists a modular maximal right ideal $M$ such that $N \subset M . K+N$ is a dense right ideal of $A$ 
by (b). Assume that $x \in K^{\perp \perp}$. Then there exists $\left\{x_{n}\right\} \subset N$ and $\left\{y_{n}\right\} \subset$ $K$ such that $x_{n}+y_{n} \rightarrow x$. Then

$$
\left|x_{n}\right|_{2}=\left|\left(x_{n}+y_{n}-x,\left.x_{n}|| x_{n}\right|_{2}\right)\right| \leqq\left|x_{n}+y_{n}-x\right|_{2} \rightarrow 0 .
$$

Therefore $y_{n} \rightarrow x$ and since $K$ is closed, $x \in K$. It follows that $K=$ $K^{\perp \perp}$. Now $K^{\perp} \subset M$, and therefore $M^{\perp} \subset K$. Since $M^{\perp}$ is a nonzero right ideal of $A, M^{\perp}=K$. Then every element in $K$ has a left adjoint by the proof of [8, Th. 4.5, p. 44]. It follows that every element in $S_{A}$ has a left adjoint, and by a similar proof every element in $S_{A}$ has a right adjoint. Then Theorem 4.1 implies that every minimal left or right ideal of $A$ is a Hilbert space in the norm determined by the inner product.

5. Algebras dual in the operator norm. A well known theorem of Bonsall and Goldie states that an annihilator $B^{*}$-algebra is dual. This was generalized by B. Yood who proved that any modular annihilator $B^{*}$-algebra is dual; see [8, Th. 4.1, p. 42]. In this section we generalize this result still further. We assume throughout that $A$ is a modular annihilator algebra with an involution ${ }^{*}$ and a norm $|\cdot|$ with the property that $\left|u^{*} u\right|=|u|^{2}$ for all $u \in A$ (such a norm always exists on $A$ when $A$ is a normed algebra and * is proper by [7, Th. 5.2, p. 358]). We call $|\cdot|$ the operator norm on $A$.

Theorem 5.1. Assume that $A$ has the properties given above. Then if every minimal left ideal of $A$ is complete in the operator norm, $A$ is dual.

We prove three lemmas.

Lemma 5.2. If every minimal left ideal of $A$ is complete in the operator norm, then there is an isometric *-representation $u \rightarrow$ $T_{u}$ of $A$ onto a subalgebra $B$ of the compact operators on a Hilbert space $\mathscr{H}$ with the following properties:

(1) $\mathscr{H}$ is the Hilbert space direct sum of a set of closed subspaces $\mathscr{H}_{\alpha}, \alpha \in I$ where $I$ is some index set.

(2) If $T \in B$, then $T$ is reduced by each $\mathscr{H}_{\alpha}, \alpha \in I$ (i.e.,

$$
T\left(\mathscr{H}_{\alpha}\right) \subset \mathscr{H}_{\alpha} \text { and } T\left(\mathscr{H}_{\alpha}^{\perp}\right) \subset \mathscr{H}_{\alpha}^{\perp}
$$

all $\alpha \in I)$.

(3) If $T \in \mathscr{F}(\mathscr{H})$ and $T$ is reduced by $\mathscr{H}_{\alpha}$ for all $\alpha \in I$, then $T \in B$.

(4) $B \cap \mathscr{F}(\mathscr{H})$ is dense in $B$.

Proof. Let $\left\{M_{\alpha} \mid \alpha \in I\right\}$ be the set of minimal two sided ideals of 
$A, I$ some index set. For each $\alpha \in I$, choose an element $h_{\alpha} \in H \cap M_{\alpha}$. Let $\mathscr{H}_{\alpha}=A h_{\alpha} . \quad A h_{\alpha}$ is an inner product space in the cannonical inner product. Also $\left|u h_{\alpha}\right|^{2}=\left|\left(u h_{\alpha}\right)^{*}\left(u h_{\alpha}\right)\right|=\left|u h_{\alpha}\right|_{2}^{2}$. Therefore $|\cdot|_{2}$ coincides with $|\cdot|$ on $A h_{\alpha}$. Therefore $\mathscr{H}_{\alpha}$ is a Hilbert space. Let $\mathscr{H}$ be the Hilbert space direct sum of the $\mathscr{H}_{\alpha}, \alpha \in I$. For each $\alpha$ we have a *-representation $u \rightarrow T_{u}^{h}{ }^{*}$ of $A$ on $A h_{\alpha}=\mathscr{H}_{\alpha} . \quad\left|T_{u}^{h}\right| \leqq$ $|u|$ for all $u \in A, \alpha \in I$. Then we define $u \rightarrow T_{u}$ a representation of $A$ on $\mathscr{C}$ in the usual fashion, $T_{u}\left(\sum_{\alpha \in I} v_{\alpha} h_{\alpha}\right)=\sum_{\alpha \in I} T_{u}^{h}\left(v_{\alpha} h_{\alpha}\right) . \quad u \rightarrow$ $T_{u}$ is a faithful *-representation of $A$ onto a subalgebra $B$ of $\mathscr{B}(\mathscr{H})$. By [1, (1.3), p. 6] $|u|=\left|T_{u}\right|$ for all $u \in A$. $T_{u}$ has finite dimensional range for all $u \in S_{A}$ by [7, Lemma 5.1, p. 358]. Also the socle of $A$ is dense in $A$ by the proof of [2, Lemma 2.6, p. 287]. It follows that $\mathscr{F}(\mathscr{H}) \cap B$ must be dense in $B$ and that $B \subset \mathscr{C}(\mathscr{H})$. It remains to prove (3). By Theorem $3.2 A$ is a left annihilator algebra, and by the proof of that theorem $\mathscr{F}\left(\mathscr{H}_{\alpha}\right) \subset\left\{T_{u}^{h_{\alpha}} \mid u \in M_{\alpha}\right\}$. Assume that $T \in \mathscr{F}(\mathscr{H}), T\left(\mathscr{H}_{\alpha}\right) \subset \mathscr{H}_{\alpha}$, and $T\left(\mathscr{H}_{\alpha}{ }^{\perp}\right) \subset \mathscr{H}_{\alpha}{ }^{1}$ for all $\alpha \in I$. Then $T\left(\mathscr{H}_{\alpha}\right)=0$ for all but a finite number of $\alpha \in I, \alpha_{1}, \alpha_{2}, \cdots, \alpha_{n}$. Then there exists $u_{k} \in M_{\alpha_{k}}, 1 \leqq k \leqq n$, such that $T_{u_{k}}^{h_{\alpha}}(x)=T(x)$ for all $x \in$ $\mathscr{H}_{\alpha}$. Let $u=u_{1}+\cdots+u_{n}$. Then $T_{u}(x)=T(x)$ for all $x \in \mathscr{H}$. This proves (3).

Lemma 5.3. Let $B$ be as in Lemma 5.2. Then $T \in \overline{T B}$ and $T \in$ $\overline{B^{\prime} T^{\prime}}$ for all $T \in B$.

Proof. Assume that $T \in B$. Then $T^{*} T$ is a compact operator on the Hilbert space $\mathscr{Z}$. Let $\left\{\lambda_{k}\right\}$ be the sequence of distinct nonzero eigenvalues of $T^{*} T$. Let $\left\{E_{k}\right\}$ be the sequence of projections onto the corresponding eigenspaces. For all $\alpha \in I$ denote by $F_{\alpha}$ the projection onto the subspace $\mathscr{H}_{\alpha}$. By hypothesis $F_{\alpha} T^{*} T=T^{*} T F_{\alpha}$ for all $\alpha \in I$. It follows that $F_{\alpha} E_{k}=E_{k} F_{\alpha}$ for all $\alpha \in I$ and all $k$. By (3) of Lemma 5.2 $E_{k} \in B$ for all $k$. Then $\left|T-\sum_{k=1}^{N} T E_{k}\right|^{2}=\mid\left(T-\sum_{k=1}^{N} T E_{k}\right)^{*}$ $\left(T-\sum_{k=1}^{N} T E_{k}\right)|=| T^{*} T-\sum_{k=1}^{N} \lambda_{k} E_{k} \mid$. Since $T^{*} T=\sum_{k=1}^{+\infty} \lambda_{k} E_{k}$ by the Spectral Theorem for compact operators, then $T\left(\sum_{k=1}^{N} E_{k}\right) \rightarrow T$ as $N \rightarrow$ $+\infty$. This proves $T \in \overline{T B}$. A similar argument using $T T^{*}$ in place of $T^{*} T$ shows that $T \in \overline{B T}$.

Lemma 5.4. Assume that $\mathscr{K}$ is a Hilbert space. Then $\mathscr{F}(\mathscr{K})$ is dual in the operator norm.

Proof. Assume that $M$ is a closed right ideal of $\mathscr{F}(\mathscr{K})$, and let $N=M+L(M)^{*} . \quad N$ is a right ideal of $\mathscr{F}(\mathscr{K})$. Let

$$
\mathscr{J}=\{T u \mid T \in N, u \in \mathscr{K}\} \text {. }
$$

As in the proof of Theorem 2.1, $\mathcal{J}$ is a subspace of $\mathscr{K}$. If $w \perp \mathcal{J}$, 
then for every $u \in \mathscr{K}$ and $T \in N,(w \mid w) T(u)=(T u, w) w=0$. Therefore $(w \mid w) N=0$. But then $(w \mid w) M=0$ and $L(M)(w \mid w)=0$. Therefore $|w|_{2}^{2}(w \mid w)=(w \mid w)^{2}=0$ so that $w=0$. This proves that $\mathscr{J}$ is dense in $\mathscr{K}$. Assume $v, w \in \mathscr{K}$. Choose $\left\{u_{n}\right\} \subset \mathscr{K}$ and $\left\{T_{n}\right\} \subset N$ such that $T_{n}\left(u_{n}\right)=v_{n} \rightarrow v$. Then $T_{n}\left(w \mid u_{n}\right)=\left(w \mid v_{n}\right) \rightarrow(w \mid v)$ so that $(w \mid v) \in$ $N$. Therefore $\mathscr{F}(\mathscr{K})=N$. Take $T \in R(L(M)) . \quad T=T_{1}+T_{2}$ where $T_{1} \in M$ and $T_{2} \in L(M)^{*}$. Then $T_{2}^{*} T=0$ and $T_{2}^{*} T_{1}=0$. Thus $T_{2}^{*} T_{2}=$ 0 which implies $T_{2}=0$. It follows that $R(L(M))=M$. If $M$ is a closed left ideal of $\mathscr{F}(\mathscr{K})$, then $L(R(M))=M$ by taking involutions. Therefore $\mathscr{F}(\mathscr{K})$ is dual.

Now we complete the proof of Theorem 5.1. By Lemma 5.2. it is enough to prove that an algebra $B$ with the properties listed in that lemma is dual. Let $F_{\alpha}$ be the projection of $\mathscr{H}$ onto $\mathscr{H}_{\alpha}$ for all $\alpha \in I$. Set $S_{\alpha}=\left\{T \in \mathscr{F}(\mathscr{H}) \mid T F_{\alpha}=F_{\alpha} T=T\right\}$. By Lemma 5.2 $S_{\alpha} \subset B$. Furthermore $\mathscr{F}\left(\mathscr{H}_{\alpha}\right)$ is isometrically isomorphic to $S_{\alpha}$. Therefore $S_{\alpha}$ is dual by Lemma 5.4. Also $S_{\alpha}$ is a two sided ideal of $B$ for each $\alpha \in I$, and $B$ is the topological sum of the $S_{\alpha}, \alpha \in I$. By Lemma 5.3 $T \in \overline{T B}$ and $T \in \overline{B T}$ for all $T \in B$. Then it follows from the proof of $[4$, Th. 2.8.29, p. 106] that $B$ is dual.

\section{REFERENCES}

1. B. Barnes, Subalgebras of modular annihilator algebras, Proc. Camb. Phil. Soc. 66 (1969), 5-12.

2. - Algebras with the spectral expansion property, Illinois J. Math. 11 (1967), 284-290.

3. I. Kaplansky, Dual rings, Ann. of Math. 49 (1948), 689-701.

4. C. Rickart, General Theory of Banach Algebras, Princeton, 1960.

5. P. P. Saworotnow, A generalization of the notion of $H^{*}$-algebra, Proc. Amer. Math. Soc. 8 (1957), 49-55.

6. B. Yood, Homomorphisms on normed algebras, Pacific J. Math. 8 (1958), 373-381.

7. - Faithful *-representations of normed algebras, Pacific J. Math. 10 (1960), 345-363.

8. - Ideals in topological rings, Canad. J. Math. 16 (1964), 28-45.

9. - On algebras which are pre-Hilbert spaces, Duke Math. J. 36 (1969), 261272.

Received January 29, 1970. This research was partially supported by a grant from the Graduate School of the University of Oregon.

The University OF OREgoN

EUGENE, OREGON 



\section{PACIFIC JOURNAL OF MATHEMATICS}

\section{EDITORS}

\author{
H. SAMELSON \\ Stanford University \\ Stanford, California 94305 \\ Richard Pierce \\ University of Washington \\ Seattle, Washington 98105
}

\author{
J. DugundJI \\ Department of Mathematics \\ University of Southern California \\ Los Angeles, California 90007 \\ RICHARD ARENS \\ University of California \\ Los Angeles, California 9.0024
}

\section{ASSOCIATE EDITORS}

\begin{tabular}{|c|c|}
\hline E. F. BECKENBACH & K. YoshidA \\
\hline \multicolumn{2}{|c|}{ SUPPORTING INSTITUTIONS } \\
\hline UNIVERSITY OF BRITISH COLUMBIA & STANFORD UNIVERSITY \\
\hline CALIFORNIA INSTITUTE OF TECHNOLOGY & UNIVERSITY OF TOKYO \\
\hline UNIVERSITY OF CALIFORNIA & UNIVERSITY OF UTAH \\
\hline MONTANA STATE UNIVERSITY & WASHINGTON STATE UNIVERSITY \\
\hline UNIVERSITY OF NEVADA & UNIVERSITY OF WASHINGTON \\
\hline NEW MEXICO STATE UNIVERSITY & * \\
\hline OREGON STATE UNIVERSITY & AMERICAN MATHEMATICAL SOCIETY \\
\hline UNIVERSITY OF OREGON & CHEVRON RESEARCH CORPORATION \\
\hline OSAKA UNIVERSITY & TRW SYSTEMS \\
\hline UNIVERSITY OF SOUTHERN CALIFORNIA & NAVAL WEAPONS CENTER \\
\hline
\end{tabular}

The Supporting Institutions listed above contribute to the cost of publication of this Journal, but they are not owners or publishers and have no responsibility for its content or policies.

Mathematical papers intended for publication in the Pacific Journal of Mathematics should be in typed form or offset-reproduced, (not dittoed), double spaced with large margins. Underline Greek letters in red, German in green, and script in blue. The first paragraph or two must be capable of being used separately as a synopsis of the entire paper. The editorial "we" must not be used in the synopsis, and items of the bibliography should not be cited there unless absolutely necessary, in which case they must be identified by author and Journal, rather than by item number. Manuscripts, in duplicate if possible, may be sent to any one of the four editors. Please classify according to the scheme of Math. Rev. Index to Vol. 39. All other communications to the editors should be addressed to the managing editor, Richard Arens, University of California, Los Angeles, California, 90024.

50 reprints are provided free for each article; additional copies may be obtained at cost in multiples of 50 .

The Pacific Journal of Mathematics is published monthly. Effective with Volume 16 the price per volume (3 numbers) is $\$ 8.00$; single issues, $\$ 3.00$. Special price for current issues to individual faculty members of supporting institutions and to individual members of the American Mathematical Society: $\$ 4.00$ per volume; single issues $\$ 1.50$. Back numbers are available.

Subscriptions, orders for back numbers, and changes of address should be sent to Pacific Journal of Mathematics, 103 Highland Boulevard, Berkeley, California, 94708.

PUBLISHED BY PACIFIC JOURNAL OF MATHEMATICS, A NON-PROFIT CORPORATION

Printed at Kokusai Bunken Insatsusha (Internatıonal Academic Printing Co., Ltd.), 7-17, Fujimi 2-chome, Chiyoda-ku, Tokyo, Japan. 


\section{Pacific Journal of Mathematics}

\section{Vol. 35, No. $3 \quad$ November, 1970}

John D. Arrison and Michael Rich, On nearly commutative degree one algebras . . . 533

Bruce Alan Barnes, Algebras with minimal left ideals which are Hilbert spaces . . . . 537

Robert F. Brown, An elementary proof of the uniqueness of the fixed point index . . . 549

Ronn L. Carpenter, Principal ideals in F-algebras .................... 559

Chen Chung Chang and Yiannis (John) Nicolas Moschovakis, The Suslin-Kleene

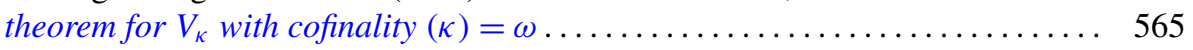

Theodore Seio Chihara, The derived set of the spectrum of a distribution

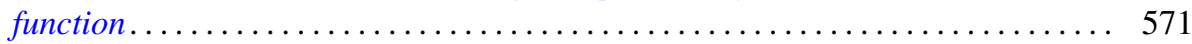

Tae Geun Cho, On the Choquet boundary for a nonclosed subspace of $C(S) \ldots \ldots \quad 575$

Richard Brian Darst, The Lebesgue decomposition, Radon-Nikodym derivative,

conditional expectation, and martingale convergence for lattices of sets .......

David E. Fields, Dimension theory in power series rings . . . . . . . . . . . .

Michael Lawrence Fredman, Congruence formulas obtained by counting

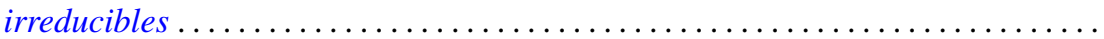

John Eric Gilbert, On the ideal structure of some algebras of analytic functions.....

G. Goss and Giovanni Viglino, Some topological properties weaker than

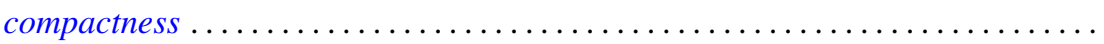

581

601

625

George Grätzer and J. Sichler, On the endomorphism semigroup (and category) of

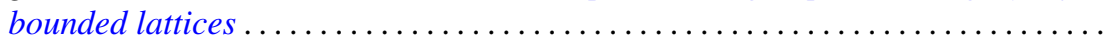

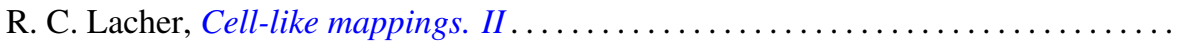

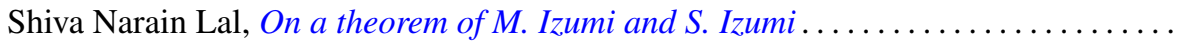

661

Howard Barrow Lambert, Differential mappings on a vector space ...............

Richard G. Levin and Takayuki Tamura, Notes on commutative power joined

semigroups.

Robert Edward Lewand and Kevin Mor McCrimmon, Macdonald's theorem for quadratic Jordan algebras.

J. A. Marti, On some types of completeness in topological vector spaces ....

Walter J. Meyer, Characterization of the Steiner point

717

Saad H. Mohamed, Rings whose homomorphic images are $q$-rings ...

727

Thomas V. O'Brien and William Lawrence Reddy, Each compact orientable surface

of positive genus admits an expansive homeomorphism ...

737

Robert James Plemmons and M. T. West, On the semigroup of binary relations...

743

Calvin R. Putnam, Unbounded inverses of hyponormal operator . .

755

William T. Reid, Some remarks on special disconjugacy criteria for differential

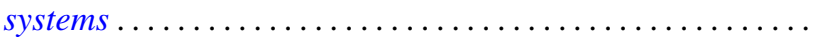

C. Ambrose Rogers, The convex generation of convex Borel sets in euclidean

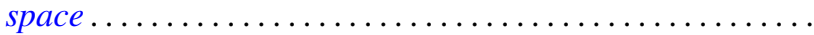

S. Saran, A general theorem for bilinear generating functions .

S. W. Smith, Cone relationships of biorthogonal systems ......

Wolmer Vasconcelos, On commutative endomorphism rings ....

795

Vernon Emil Zander, Products of finitely additive set functions from Orlicz

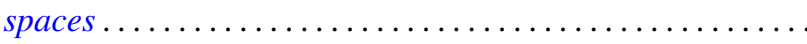

G. Sankaranarayanan and C. Suyambulingom, Correction to: "Some renewal

theorems concerning a sequence of correlated random variables" .

Joseph Zaks, Correction to: "Trivially extending decompositions of $E^{n}$ ”....... 805

Dong Hoon Lee, Correction to: "The adjoint group of Lie groups" ............ 805

James Edward Ward, Correction to: "Two-groups and Jordan algebras". 